\title{
Controlling the rotation modes of hematite nanospindles by dynamic magnetic fields
}

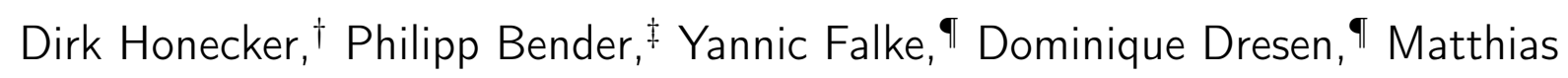

Kundt, Annette Schmidt, Andreas Tschöpe, ${ }^{\S}$ Michael Sztucki, " Manfred

$$
\text { Burghammer, "l and Sabrina Disch*, }
$$

$\dagger$ †SIS Neutron and Muon Facility, Rutherford Appleton Laboratory, Didcot, OX11 0QX,UK

$\ddagger$ Heinz Maier-Leibnitz Zentrum (MLZ), Technische Universität München,

Lichtenbergstraße 1, 85748 Garching, Germany

\Department für Chemie, Universität zu Köln, Greinstr. 4-6, 50939 Köln, Germany

$\S$ Experimentalphysik, Universität des Saarlandes, Saarbrücken, Germany

||European Synchrotron Radiation Facility (ESRF), F-38043 Grenoble, France

E-mail: sabrina.disch@uni-koeln.de

\begin{abstract}
The magnetic field-induced actuation of colloidal nanoparticles has enabled tremendous recent progress towards microrobots, suitable for a variety of applications including targeted drug delivery, environmental remediation or minimally invasive surgery. Further size reduction to the nanoscale requires enhanced control of orientation and locomotion to overcome dominating viscous properties.

Here we demonstrate how the coherent precession of nanoscale hematite spindles can be controlled via dynamic magnetic field. Using time-resolved Small-Angle Scattering and optical transmission measurements, we reveal a clear frequency-dependent variation of orientation and rotation of an entire ensemble of hematite nanospindles. Our findings
\end{abstract}


are in line with the different motion mechanisms observed for much larger, micron sized elongated particles near surfaces. The different dynamic rotation modes promise hematite nanospindles as a suitable model system towards field-induced locomotion in nanoscale magnetic robots.

\section{Introduction}

Field-driven actuation on magnetic particles builds the foundation of various intriguing applications, including self-propelling particles in active matter, ${ }^{1,2}$ mixers in microfluidics, ${ }^{3}$ and viscosity probes in nanorheology, ${ }^{4}$ but also aspects based on structure formation, such as field-induced self-organization ${ }^{5-7}$ and smart fluids. ${ }^{8,9}$ Biological microorganisms such as magnetotactic bacteria are inspiring model systems for magnetic microswimmers ${ }^{10}$ operating at low Reynolds numbers, but also large enough so that their movement and dynamics can be tracked by optical techniques. The development of artificial magnetically-driven micro- and nanorobots has received tremendous attention in the last few years, ${ }^{2,11,12}$ leading to advanced applications including magnetic two-arm nanoswimmers ${ }^{13}$ or the combination of magnetic hyperthermia and magnetically driven propulsion for local pollutant remediation. ${ }^{14}$

Achieving a remotely controlled, sustained translational motion for active Brownian particles in a viscous fluid is a challenging endeavour. This is typically approached by advanced synthesis of complex, chiral structures, ${ }^{15}$ such as helical structures for magnetically actuated propulsion in dynamic, rotating or oscillating magnetic fields. ${ }^{16}$ Very recently, a rich variety of motion mechanisms has been achieved using achiral, elongated objects with perpendicular magnetization. ${ }^{17-19}$ Even random shapes based on nanoparticle aggregates have been shown to succeed as fast magnetic micropropellers. ${ }^{20}$ All these particles propel when stirred by a relatively weak rotating uniform magnetic field (of the order of a few $\mathrm{mT}$ ). The motion mechanism is tunable from tumbling through precessing to rolling motion via the frequency of the rotating magnetic field, ${ }^{19}$ ultimately fading into the step-out behavior associated with declining propulsion velocity at highest frequencies. ${ }^{21}$ These examples emphasize the great 
potential of dynamic magnetic fields for the controlled locomotion of mesoscopic magnetic particles in a viscous medium and the design of magnetoresponsive soft matter.

If the particle size is reduced to the nanoscale, thermal effects dominate, and stochastic motion of the particles due to random collisions (i.e., Brownian motion) complicate a controlled steering of magnetic nanoparticles in viscous fluids. ${ }^{22}$ One of the rare examples of successfully synthesized propellers with dimensions below $1 \mu \mathrm{m}$ was demonstrated with carboncoated aggregates of nanoparticles involving a post-synthesis selection process. ${ }^{23}$ Hematite nanospindles represent a peculiar case of anisometric nanoparticles that promise to progress magnetohydrodynamics to an even smaller length scale. Hematite nanospindles are achiral, elongated nanoparticles (i.e., particles with at least two dimensions between 1-100 nm) that intrinsically exhibit a magnetization perpendicular to the long axis. This geometry is similar to the microwires with artificially engineered perpendicular magnetization ${ }^{17,19}$ and therefore promising for locomotion of nanoobjects in dynamic magnetic fields. As the particle moments align parallel with an applied magnetic field, the hematite nanospindles orient with their long spindle axis perpendicular to a static field direction. ${ }^{24,25}$ Within this plane perpendicular to the field the spindle orientation fluctuates randomly. There are two different characteristic relaxation frequencies associated with (1) a rotation of the spindle along the long rotational axis and (2) a rotation along the minor axes.

Here we demonstrate that by application of a rotating magnetic field in a suitable frequency range, the dynamic reorientation of hematite nanospindles can be forced into a synchronized spinning. Using time-resolved small-angle X-ray scattering (SAXS), we reveal how the orientation of an ensemble of water-dispersed hematite nanospindles can be tuned between a coherent precession and a collinear alignment by suppression of one of the two distinct rotation directions. The time-resolution of stroboscopic SAXS provides information on the dynamic particle orientation while applying alternating or rotating magnetic field. Our results are supported with complimentary optical transmission measurements and agree with theoretical estimates of the rotational diffusion constant. The observations demonstrate 
that hematite nanospindle hold out the key features for future application as nanopropellers.

\section{Results and Discussion}

To achieve well-separated relaxation time scales, a large aspect ratio of the hematite spindles is required. We therefore synthesized hematite nanospindles according to the hydrolysis method developed by Matijević and co-workers that allows to tune the aspect ratio. ${ }^{26}$ TEM analysis reveals an average diameter of $51.5 \mathrm{~nm}$ and a particle length of $374 \mathrm{~nm}$ along with normal size distributions in the range of $13-20 \%$, corresponding to an aspect ratio of around 7.3 (Figure S1). Small-angle X-ray scattering (SAXS) of the colloidal suspension of nanospindles in water, performed at the instrument ID13 (ESRF), confirms the average particle diameter of $49.9 \mathrm{~nm}$ with an intermediate lognormal size distribution of $9.6 \%$ (Figure S5). The quasi-static magnetization measurement of the colloidal suspension further confirms the weakly ferromagnetic behavior of hematite above the Morin transition by the pseudo-superparamagnetic field dependence typically observed for magnetically blocked nanoparticles in dispersion (Figure S2). Refinement of the Langevin behavior reveals an integral nanoparticle moment of $7.643 \cdot 10^{-19} \mathrm{~J} / \mathrm{T}$, corresponding to $82414(705) \mu_{B}$. Along with the morphological particle volume of $5.19 \cdot 10^{-22} \mathrm{~m}^{3}$ as determined from TEM and SAXS analysis, a spontaneous magnetization of $1473 \mathrm{~A} / \mathrm{m}$ is derived. The obtained spontaneous magnetization represents only $74 \%$ of the bulk value for hematite of $\sim 2000 \mathrm{~A} / \mathrm{m},{ }^{27}$ in agreement with earlier reports. ${ }^{24,28}$ From this follows that the particles are not interacting and hence can freely rotate in suspension when applying external magnetic fields.

The anisotropy of the relaxation frequencies is confirmed by depolarized dynamic light scattering (DDLS, Fig. S3) and AC magnetic susceptometry (ACMS, Fig. S4). The rotational diffusion constant $D_{\mathrm{R}}=149(7) \mathrm{s}^{-1}$ derived from DDLS analysis agrees well with the

theoretical estimate of $179 \mathrm{~s}^{-1}$ for an ellipsoid of revolution of the same dimensions ${ }^{29,30}$ and corresponds to a characteristic frequency of $\nu_{\perp}=D_{\mathrm{R}} / \pi=47(2) \mathrm{Hz}$. In contrast, ACMS 
reveals a characteristic frequency of $\nu_{\mathrm{ACMS}}=278 \mathrm{~Hz}$. The strong difference between these characteristic frequencies highlights the different mechanisms probed by both techniques. Whereas DDLS is sensitive to the orientational diffusion of the particles in dilute dispersion, and hence can only sense the rotation around the minor spindle axis, ACMS probes the fieldinduced orientation of the spindle magnetization, including contributions of both rotation around the minor and principal axes. The characteristic frequency for rotation around the principal spindle axis is therefore not unambiguously accessible from ACMS, and will lie well beyond $\nu_{\mathrm{ACMS}}=278 \mathrm{~Hz}$. The significant difference between the characteristic frequencies for rotation around principal and minor spindle axes is an important prerequisite for a tunable motion control: we expect that for a magnetic field rotating with a frequency between both characteristic frequencies, a transition between coherent precession and synchronized spinning of nanospindles in dispersion is achievable. ${ }^{31}$

Small-angle scattering probes nanoscale inhomogeneities with suitable spatial resolution to address the orientation distribution of a nanoparticle ensemble, ${ }^{25,32,33}$ whereas timeresolved approaches give versatile opportunities to study in-situ nanoparticle ensemble dynamics. ${ }^{34-36}$ Stroboscopic SAXS provides the necessary time resolution to monitor changes in the orientation distribution near the characteristic frequencies. We therefore performed time-resolved SAXS at the microfocus beamline ID13 (ESRF) to monitor the dynamic fielddependent orientation of hematite spindles driven by a custom-made set of Helmholtz coils. The two Helmholtz coils can each generate alternating magnetic fields, and together a magnetic field rotating in the horizontal plane when both applied fields have the same amplitude and frequency with a $90^{\circ}$ phase shift (Fig. 1a). The magnetic field period was divided into 20 frames by synchronizing the coil setup with the detector to obtain the time-resolved scattering patterns as shown in Figure 1f. The Maxipix detector allowed to measure stroboscopically up to $300 \mathrm{~Hz}$. To emphasize the scattering anisotropy caused by the particle alignment in the applied field, all the following scattering images will be the difference pattern with respect to the isotropic scattering pattern in zero field (see Fig. S6). 

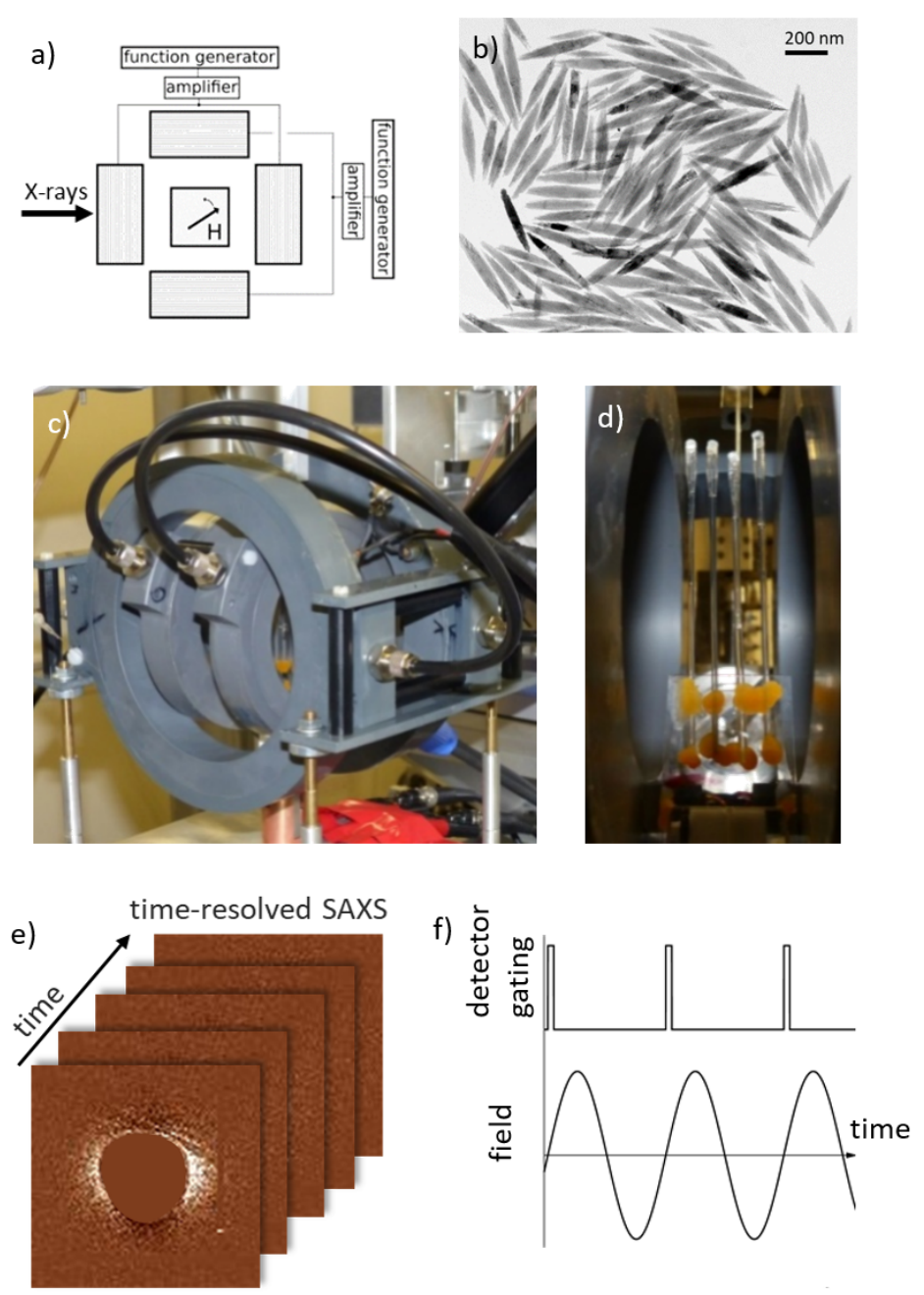

Figure 1: Experimental setup for stroboscopic SAXS at the ID13 beamline (ESRF). a) Schematic drawing of two Helmholtz coils placed parallel and perpendicular to the X-ray beam. b) TEM by hematite nanospindles. c) Helmholtz coil set. d) Colloidal dispersion of nanospindles and reference samples, mounted in quartz capillaries in the center of the field setup. e) Time-resolved acquisition of SAXS. Detector images show a large beam stop in the center, surrounded by the SAXS signal. Detector images are presented as the difference scattering pattern with respect to the isotropic scattering pattern in zero field. f) Detector gating with respect to the magnetic field phase. 


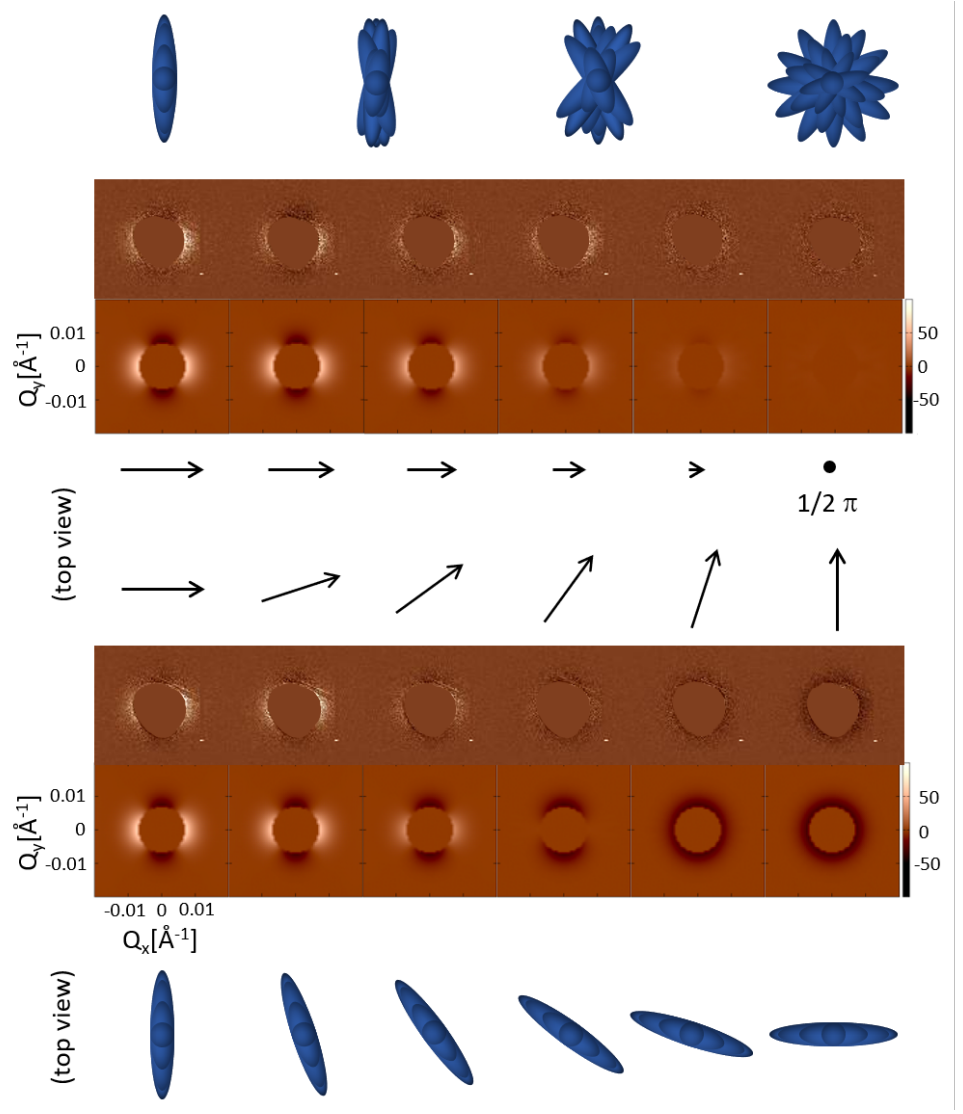

Figure 2: Stroboscopic SAXS by hematite nanospindles in alternating (top) and rotating (bottom) magnetic fields of $25 \mathrm{~Hz}$. Field configuration is depicted by black arrows (center). The SAXS difference detector images are shown for a quarter period $(0-1 / 2 \pi)$. Computed scattering patterns according to the expected orientation of the nanospindle ensemble are shown below the respective data. The average, time-resolved nanospindle orientation is depicted schematically. 
At low frequencies of $25 \mathrm{~Hz}$, where the moments of the entire nanospindle ensemble follow the applied field, a periodic variation of the anisotropic scattering intensities is observed for both alternating and rotating magnetic fields (Fig. 2, full period shown in Fig. S7). In alternating magnetic field, the time-resolved variation of scattering intensities indicates a dynamic interplay of order and disorder. Whereas the SAXS intensity is isotropic at times near the zero-field condition $(1 / 2 \pi$ and $3 / 2 \pi)$, corresponding to the random nanoparticle orientation in the absence of a magnetic field, a clear scattering anisotropy is observed at maximum field of $10 \mathrm{mT}$ ( 0 and $\pi$ ). This scattering anisotropy results from the orientation of the spindles perpendicular to the inducing field, where the degree of alignment corresponds well to the Langevin parameter of 1.85, estimated for the integral nanospindle moment in the maximum applied field of $10 \mathrm{mT}$. We confirm this by computing the expected scattering pattern for a spindle ensemble using the spindle dimensions, integral particle moment, and average applied field for each frame according to the Boltzmann statistics of the particle distribution. ${ }^{25}$ As shown in Fig. 2, the computed scattering pattern is in excellent agreement with the measured pattern. In case of a rotating magnetic field of $10 \mathrm{mT}$, the easy magnetic axes of the nanospindles will maintain their orientation towards the applied field and the nanoparticle ensemble may fulfill a complete turn within one period of the applied field as long as thermal motion and fluid friction are subsidiary effects. We identify this behavior in low frequency rotating magnetic field as coherent precession: the spindle ensemble fulfills precession around the field normal with a coherent phase behavior, albeit different precession angles. The difference between the two cases of alternating and rotating magnetic fields becomes very clear at the $1 / 2 \pi$ and $3 / 2 \pi$ time frames. In these time frames the applied rotating field is oriented parallel to the X-ray beam, resulting in isotropic scattering patterns. However, the difference of the scattering intensities against the zero-field state is negative. The computed scattering pattern (Fig. 2) confirms that the nanospindle ensemble is oriented parallel to the detector plane, whereas for the alternating field these time frames correspond to an isotropic, disordered ensemble at nearly zero field and hence vanishing 
difference scattering intensities.

a) alternating magnetic field

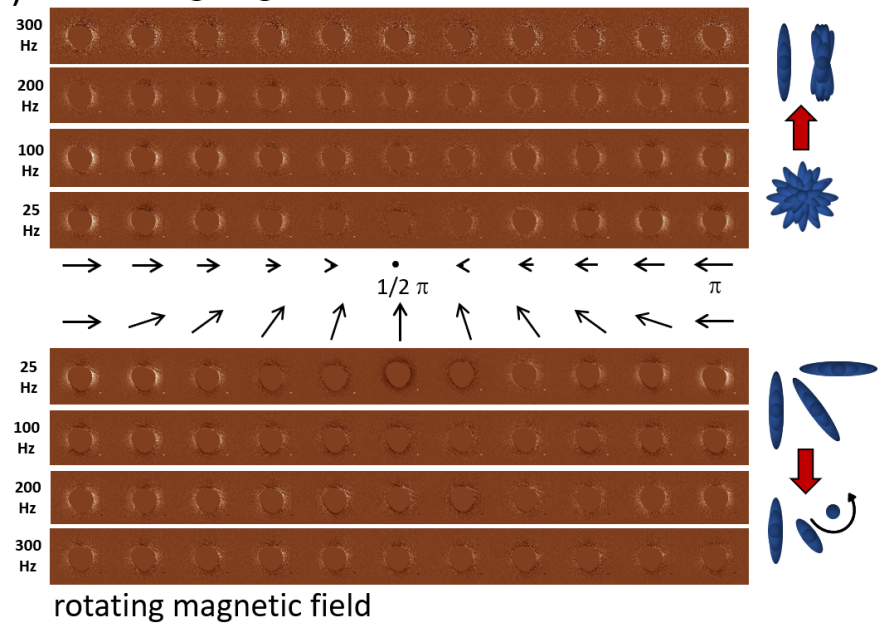

b)
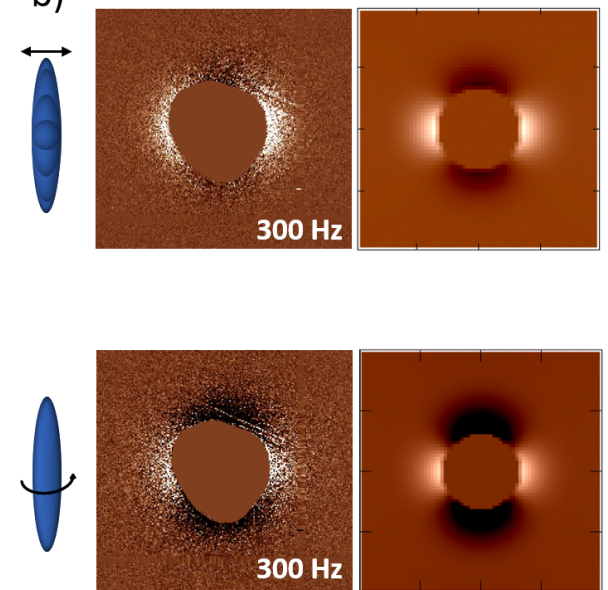

Figure 3: a) Stroboscopic SAXS data by hematite nanospindles in alternating (top) and rotating (bottom) magnetic fields up to $300 \mathrm{~Hz}$. Field configuration is depicted by black arrows (center) next to the SAXS difference detector images for half a period. b) Timeintegrated SAXS for alternating (top) and rotating (bottom) magnetic field with $300 \mathrm{~Hz}$ (left) along with computed scattering patterns (right).

The evolution of difference scattering patterns with increasing frequency for both the alternating and rotating field (Fig. 3) reveals how the time-dependent fluctuation of the scattering intensities disappears, while the scattering anisotropy remains. This is a clear signal of a transition from the dynamic particle reorientation observed at low frequency towards a confined particle arrangement. A more quantitative picture is established by analysis of the time- and frequency-dependent scattering anisotropy of the two field configurations (Fig. 4a and 4b), derived as the difference between scattering intensity in horizontal and vertical directions (see SI). Whereas in the low frequency case $(25 \mathrm{~Hz}$ ) a clear time-resolved variation between maximum and vanishing scattering anisotropies is evident, the increasing magnetic field frequency is accompanied with a significant phase lag of the scattering anisotropy. This is a strong indication that there is a dissipative process acting such that the nanospindles cannot follow the dynamic magnetic field anymore at elevated frequencies. The time-dependent amplitude in scattering anisotropy decreases, corresponding to a more 
and more static orientation of the ensemble of nanospindles. However, there is a significant scattering anisotropy even at the highest investigated stroboscopic frequency of $300 \mathrm{~Hz}$ at all times. This indicates that the average orientation of a significant portion of the nanospindle ensemble is not isotropic.

For higher frequencies beyond $300 \mathrm{~Hz}$, only time-averaged scattering anisotropies, corresponding to the dotted lines in Fig. $4 \mathrm{a}$ and $\mathrm{b}$, are accessible from time-averaged SAXS data. Over the complete frequency range, these illustrate the transitions between different types of collective motion (Fig. 4c). A maximal scattering anisotropy occurs for 150-200 $\mathrm{Hz}$, i.e. in between the characteristic frequencies for rotation around the minor axis $(47 \mathrm{~Hz}$ as determined from DDLS) and principal axis (beyond $278 \mathrm{~Hz}$ as estimated from ACMS and DDLS). In this frequency range, rotation around the principal axis is still allowed while rotation around the minor axis is inhibited. Beyond the characteristic frequencies for rotation around both principal and minor axes, the spindles do not follow the field variation anymore. Consequently, the scattering anisotropy decreases continuously, indicating an increasingly isotropic orientation of the nanoparticle ensemble.

Despite the similar behavior of the scattering anisotropy with dynamic field frequency, a different spindle orientation distribution for alternating and rotating magnetic fields at intermediate frequency is inferred from the scattering intensities shown in Fig. 3b. The differential scattering patterns correspond well to those computed for orientation distributions with the long spindle axis either confined to the plane perpendicular to the alternating magnetic field or to the direction perpendicular to the rotating field plane (Fig. 3b). In case of the alternating field, the time-independent but anisotropic scattering intensity indicates that the spindle long axes stabilize permanently in the plane perpendicular to the field direction. We understand this such that beyond the characteristic frequency for rotation around the minor axis, the average field-induced angular moment becomes larger than the disordering Brownian momentum. In effect, the spindles become confined in the plane perpendicular to the field and fulfill random rotation on this $2 \mathrm{D}$ plane. ${ }^{37}$ In the rotating 
case, on the other hand, a particle experiences a constant torque produced by the rotating magnetic field that increases with frequency and eventually becomes significantly larger than Brownian random fluctuation within the 2D plane. A self-stabilizing rotational motion is favored as the viscous friction is reduced in a rotation around the long axis as compared to precessing motion involving rotation around the short axis. As a result, the spindle long axis stabilizes permanently in the direction perpendicular to the rotating field plane such that the magnetic moments can follow the rotating field on the shortest path of least action. We understand this as a synchronized spinning: the ensemble of collinearly aligned nanospindles rotates synchronously around their major spindle axes.
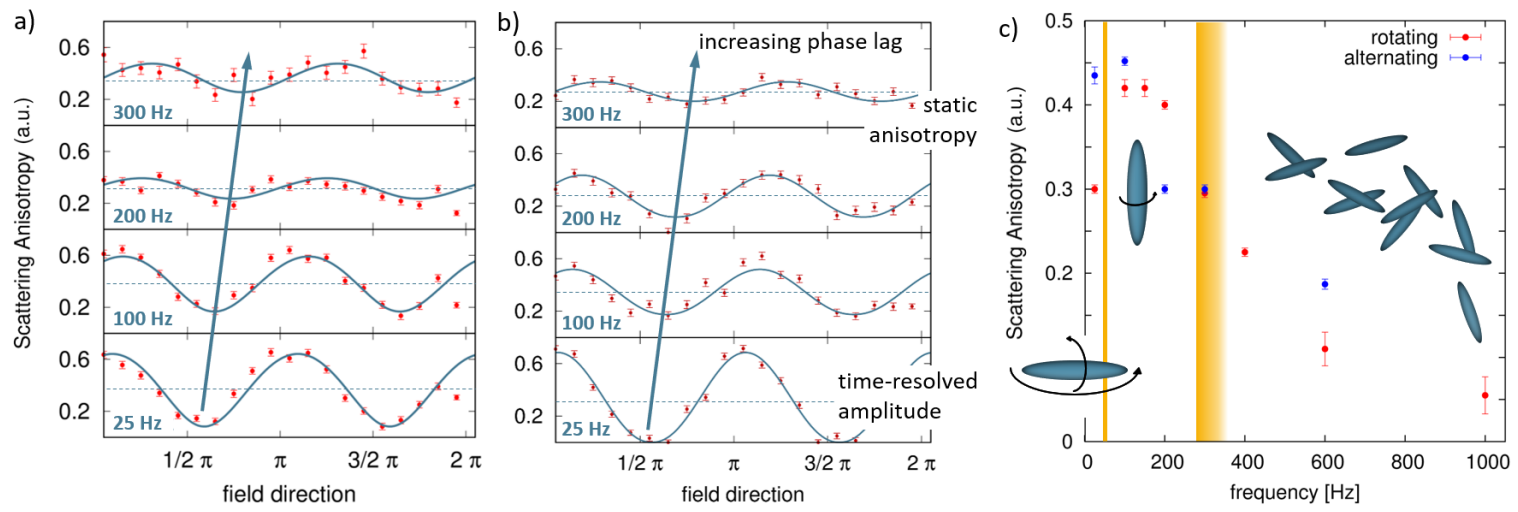

Figure 4: Time-resolved SAXS by hematite nanospindles in dynamic magnetic field with varying frequencies. Extracted scattering anisotropies for all time frames measured at the different frequencies of the a) alternating and b) rotating magnetic field. c) Frequencydependent scattering anisotropy derived from time-averaged SAXS measurements in alternating (blue) and rotating (red) field. The characteristic frequencies for rotation around the minor $(47(2) \mathrm{Hz}$ ) and major axis (beyond $278 \mathrm{~Hz}$ ) are indicated by orange bars.

The field-induced, frequency-dependent reorientation variation observed by time-resolved SAXS is strongly supported by optical transmission measurements (Fig. 5). The optical transmission of linearly polarized laser light through a dilute suspension of elongated nanoparticles depends directly on the relative orientation of laser light polarization and principal nanoparticle axis. For hematite nanospindles in a static magnetic field, an increase in optical transmission with applied static field parallel to the polarization direction and a 

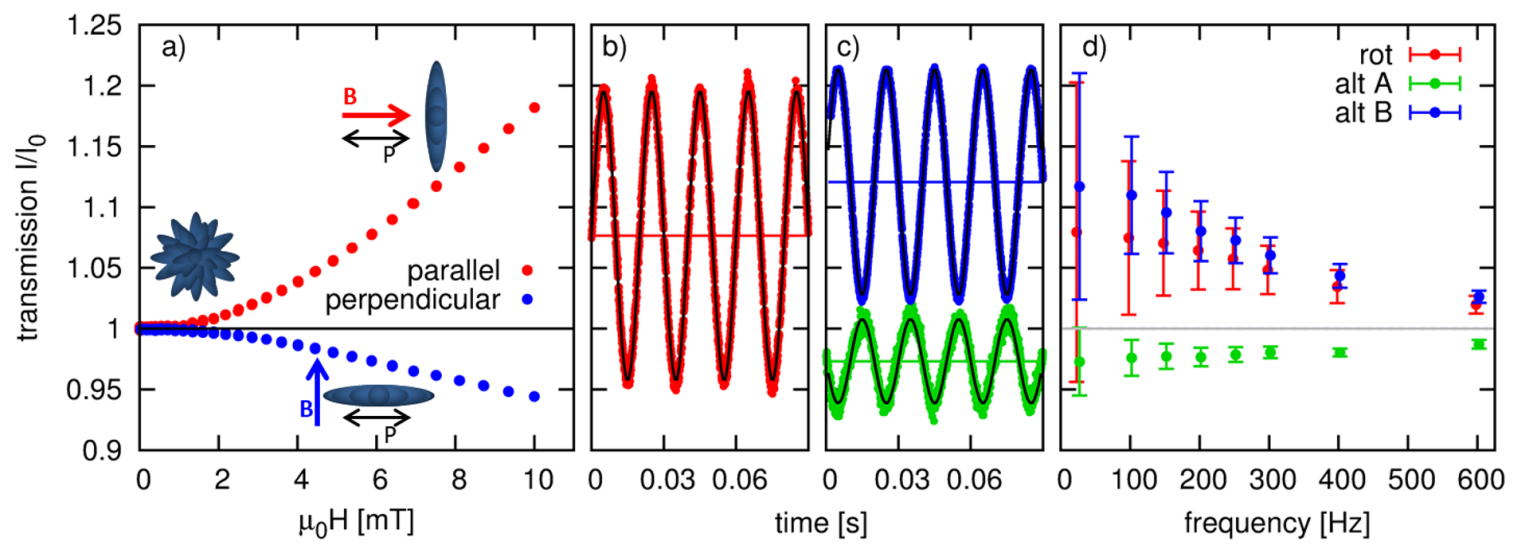

Figure 5: Optical transmission measurements: a) field-dependent relative optical transmission in DC magnetic field along with time-resolved optical transmission for (b) rotating and (c) alternating magnetic fields of $25 \mathrm{~Hz}$ and $10 \mathrm{mT}$, d) frequency dependence of the relative optical transmission $(\bullet)$ with error bars representing the time-resolved amplitude.

decrease in optical transmission for a magnetic field applied perpendicular to the polarization direction is consequently observed (Fig. 5a). The time-resolved optical transmission recorded in rotating magnetic field of $25 \mathrm{~Hz}$ (Fig. 5b) oscillates exactly between the maximum and minimum static transmission, confirming that the entire nanoparticle ensemble follows the applied field. Similarly, in alternating magnetic field of $25 \mathrm{~Hz}$ (Fig. 5c) the timeresolved optical transmission oscillates between the transmission extrema and 1, indicating considerable orientation of the nanospindles in the maximum applied field and isotropic orientation in zero field. With increasing frequency of both rotating and alternating magnetic field (Fig. 5d), the full orientational order of the static or nearly-static case is not reached anymore. This is indicated by the decrease of the time-resolved amplitudes as well as by the center of the relative optical transmission approaching 1. However, the time-averaged anisotropy remains high, so that the full rotation amplitudes move above the isotropic case $\left(I / I_{0}=1\right.$, grey line in Fig. $\left.5 \mathrm{~d}\right)$, indicating that a new, ordered state is achieved. The baseline of the rotation amplitude above 1 indicates that in the characteristic frequency range (with a maximum at 200-250 Hz), a significant portion of the spindles must be aligned perpendicular to the polarization direction at all times, which can only be the case if the spindles are 
aligned perpendicular to the rotating field plane. Likewise, the oscillation amplitudes in the alternating field do not reach the fully isotropic state at elevated frequencies, supporting the anisotropic nanospindle orientation observed with SAXS.

To summarize the response of the nanospindle ensemble in rotating fields: at low frequencies below the characteristic relaxation frequencies, the nanospindles follow the magnetic field and remain quasi-statically oriented with their major axis perpendicular to the inducing magnetic field, corresponding to coherent precession around the field normal. For intermediate magnetic field frequencies between the two relaxation time scales, rotation around the minor particle axis becomes suppressed, resulting in a decreasing precession angle. In consequence, the particles are driven into a collinearly aligned orientation of the major particle axis with synchronized spinning around the normal to the rotation plane. At high frequencies beyond the characteristic frequencies of rotation around both axes, the spindle ensemble disorients toward isotropic disorder.

\section{Conclusion}

We have elucidated the frequency-dependent reorientation behavior of hematite nanospindles in dynamic magnetic fields. The study emphasizes the potential of dynamic fields to control the rotation modes of shape anisotropic colloidal magnetic nanoparticles with perpendicular magnetic anisotropy.

Stroboscopic SAXS resolves signatures of different types of motion with a clear enhancement of the particle orientation in the intermediate frequency range between the characteristic relaxation time scales. The orientation behavior strikingly differs between alternating or rotating magnetic field. Time-resolved SAXS is a valuable tool to investigate this type of dynamic self-organization in-situ towards nearly collinear alignment of nanospindles perpendicular to the rotating magnetic field. The peculiar behavior in rotating magnetic field from coherent precession to synchronized spinning is understood similar to the frequency 
dependent variation of motion mechanisms of individual microwires near a surface boundary, ranging from tumbling and precessing to a rolling motion with increasing frequency. ${ }^{19}$ With further increasing frequency, the effective field that aligns the particle upwards reduces due to the increasing phase lag of the spindle magnetization towards the rotating magnetic field. For dynamic frequencies well above the characteristic frequencies for rotation around the major axis, the synchronized rotational motion of the spindle ensemble ceases. This corresponds well to the step-out behavior observed as a decay in propulsion velocity of helical objects in rotating field with increasing frequency. ${ }^{21,38}$ The characteristic frequencies for rotational diffusion of hematite spindles are adjustable by variation of the spindle length and aspect ratio through synthetic considerations. This will enable direct tuning of the frequency range needed to control the different rotation modes. The ability to control the dynamic reorientation of large ensembles (in the order of $10^{10}$ ) nanoscale magnetic particles establishes an important step towards field-driven actuation and locomotion. With this prerequisite, oriented locomotion of a swarm of nanoparticles may become accessible using complex magnetic field geometries, such as combined rotating and static magnetic fields. ${ }^{39}$

\section{Acknowledgement}

We acknowledge the ESRF for the provision of synchrotron radiation facilities at the Microfocus beamline ID13. This work benefited from the use of the SasView application, originally developed under NSF award DMR-0520547. SasView contains code developed with funding from the European Union's Horizon 2020 research and innovation programme under the SINE2020 project, grant agreement No 654000. We thank Stefan Roitsch for the acquisition of TEM micrographs. Financial support from the German Research Foundation (DFG: Emmy Noether Grant DI 1788/2-1) is gratefully acknowledged. 


\section{Supporting Information Available}

The following files are available free of charge.

- Details on the nanoparticle synthesis and characterization using electron microscopy, vibrating sample magnetometry, depolarized dynamic light scattering, AC magnetic susceptometry, and optical transmission measurements, and the time-resolved SAXS experiment.

\section{References}

(1) Lum, G. Z.; Ye, Z.; Dong, X.; Marvi, H.; Erin, O.; Hu, W.; Sitti, M. ShapeProgrammable Magnetic Soft Matter. PNAS 2016, 113, E6007-E6015.

(2) Zhou, H.; Mayorga-Martinez, C. C.; Pané, S.; Zhang, L.; Pumera, M. Magnetically Driven Micro and Nanorobots. Chemical Reviews 2021, 121, 4999-5041, PMID: 33787235.

(3) Nguyen, N.-T. Micro-Magnetofluidics: Interactions between Magnetism and Fluid Flow on the Microscale. Microfluid Nanofluid 2012, 12, 1-16.

(4) Calero-DdelC, V. L.; Santiago-Quiñonez, D. I.; Rinaldi, C. Quantitative Nanoscale Viscosity Measurements Using Magnetic Nanoparticles and SQUID AC Susceptibility Measurements. Soft Matter 2011, 7, 4497.

(5) Disch, S.; Wetterskog, E.; Hermann, R. P.; Salazar-Alvarez, G.; Busch, P.; Brückel, T.; Bergström, L.; Kamali, S. Shape Induced Symmetry in Self-Assembled Mesocrystals of Iron Oxide Nanocubes. Nano Lett. 2011, 11, 1651-1656.

(6) Wetterskog, E.; Klapper, A.; Disch, S.; Josten, E.; Hermann, R. P.; Rücker, U.; Brückel, T.; Bergström, L.; Salazar-Alvarez, G. Tuning the Structure and Habit of Iron Oxide Mesocrystals. Nanoscale 2016, 8, 15571-15580. 
(7) Kapuscinski, M.; Munier, P.; Segad, M.; Bergström, L. Two-Stage Assembly of Mesocrystal Fibers with Tunable Diameters in Weak Magnetic Fields. Nano Letters 2020, 20, 7359-7366, PMID: 32924498.

(8) Stanway, R. Smart Fluids: Current and Future Developments. Materials Science and Technology 2004, 20, 931-939.

(9) de Vicente, J.; Klingenberg, D. J.; Hidalgo-Alvarez, R. Magnetorheological Fluids: A Review. Soft Matter 2011, 7, 3701.

(10) Bente, K.; Codutti, A.; Bachmann, F.; Faivre, D. Biohybrid and Bioinspired Magnetic Microswimmers. Small 2018, 25.

(11) Chen, X.-Z.; Jang, B.; Ahmed, D.; Hu, C.; Marco, C. D.; Hoop, M.; Mushtaq, F.; Nelson, B. J.; Pané, S. Small-Scale Machines Driven by External Power Sources. Adv. Mater. 2018, 23.

(12) Cao, Q. Recent Advances in Manipulation of Micro- and Nano-Objects with Magnetic Fields at Small Scales. Materials Horizons 2020, 29.

(13) Li, T.; Li, J.; Morozov, K. I.; Wu, Z.; Xu, T.; Rozen, I.; Leshansky, A. M.; Li, L.; Wang, J. Highly Efficient Freestyle Magnetic Nanoswimmer. Nano Lett. 2017, 7.

(14) Dhar, P.; Narendren, S.; Gaur, S. S.; Sharma, S.; Kumar, A.; Katiyar, V. Self-Propelled Cellulose Nanocrystal Based Catalytic Nanomotors for Targeted Hyperthermia and Pollutant Remediation Applications. International Journal of Biological Macromolecules 2020, 158, 1020-1036.

(15) Tierno, P. Recent advances in anisotropic magnetic colloids: realization, assembly and applications. Physical chemistry chemical physics 2014, 16, 23515-23528.

(16) Mandal, P.; Patil, G.; Kakoty, H.; Ghosh, A. Magnetic Active Matter Based on Helical Propulsion. Acc. Chem. Res. 2018, 51, 2689-2698. 
(17) Mair, L. O.; Evans, B. A.; Nacev, A.; Stepanov, P. Y.; Hilaman, R.; Chowdhury, S.; Jafari, S.; Wang, W.; Shapiro, B.; Weinberg, I. N. Magnetic Microkayaks: Propulsion of Microrods Precessing near a Surface by Kilohertz Frequency, Rotating Magnetic Fields. Nanoscale 2017, 9, 3375-3381.

(18) Martinez-Pedrero, F.; Navarro-Argemí, E.; Ortiz-Ambriz, A.; Pagonabarraga, I.; Tierno, P. Emergent hydrodynamic bound states between magnetically powered micropropellers. Science advances 2018, 4, eaap9379.

(19) Jang, B.; Hong, A.; Alcantara, C.; Chatzipirpiridis, G.; Martí, X.; Pellicer, E.; Sort, J.; Harduf, Y.; Or, Y.; Nelson, B. J.; Pané, S. Programmable Locomotion Mechanisms of Nanowires with Semihard Magnetic Properties Near a Surface Boundary. ACS Appl. Mater. Interfaces 2019, 11, 3214-3223.

(20) Vach, P. J.; Fratzl, P.; Klumpp, S.; Faivre, D. Fast Magnetic Micropropellers with Random Shapes. Nano Lett. 2015, 15, 7064-7070.

(21) Bachmann, F.; Giltinan, J.; Codutti, A. Opportunities and Utilization of Branching Andstep-out Behavior in Magnetic Microswimmers with a Nonlinear Response. Applied Physics Letters 2021, 7.

(22) Jang, S. P.; Choi, S. U. Role of Brownian motion in the enhanced thermal conductivity of nanofluids. Applied physics letters 2004, 84, 4316-4318.

(23) Vach, P. J.; Brun, N.; Bennet, M.; Bertinetti, L.; Widdrat, M.; Baumgartner, J.; Klumpp, S.; Fratzl, P.; Faivre, D. Selecting for Function: Solution Synthesis of Magnetic Nanopropellers. Nano Lett. 2013, 13, 5373-5378.

(24) Reufer, M.; Dietsch, H.; Gasser, U.; Grobety, B.; Hirt, A. M.; Malik, V. K.; Schurtenberger, P. Magnetic Properties of Silica Coated Spindle-Type Hematite Particles. J. Phys.: Condens. Matter 2011, 23, 065102. 
(25) Zákutná, D.; Falke, Y.; Dresen, D.; Prévost, S.; Bender, P.; Honecker, D.; Disch, S. Morphological and Crystallographic Orientation of Hematite Spindles in an Applied Magnetic Field. Nanoscale 2019, 11, 7149-7156.

(26) Ozaki, M.; Kratohvil, S.; Matijević, E. Formation of Monodispersed Spindle-Type Hematite Particles. Journal of Colloid and Interface Science 1984, 102, 146-151.

(27) Bødker, F.; Hansen, M. F.; Koch, C. B.; Lefmann, K.; Mørup, S. Magnetic Properties of Hematite Nanoparticles. Phys. Rev. B 2000, 61, 6826-6838.

(28) Hoffelner, D.; Kundt, M.; Schmidt, A. M.; Kentzinger, E.; Bender, P.; Disch, S. Directing the Orientational Alignment of Anisotropic Magnetic Nanoparticles Using Dynamic Magnetic Fields. Faraday Discuss. 2015, 181, 449-461.

(29) Perrin, F. Brownian motion of an ellipsoid - I. Dielectric dispersion for ellipsoidal molecules. J. Phys. Radium 1934, 5, $497-511$.

(30) Dubin, S. B.; Clark, N. A.; Benedek, G. B. Measurement of the Rotational Diffusion Coefficient of Lysozyme by Depolarized Light Scattering: Configuration of Lysozyme in Solution. The Journal of Chemical Physics 1971, 54, 5158-5164.

(31) Ghosh, A.; Dasgupta, D.; Pal, M.; Morozov, K. I.; Leshansky, A. M.; Ghosh, A. Helical Nanomachines as Mobile Viscometers. Advanced Functional Materials 2018, 28, 1705687.

(32) Li, T.; Senesi, A. J.; Lee, B. Small angle X-ray scattering for nanoparticle research. Chem. Rev. 2016, 116, 11128-11180.

(33) Jeffries, C. M.; Ilavsky, J.; Martel, A.; Hinrichs, S.; Meyer, A.; Pedersen, J. S.; Sokolova, A. V.; Svergun, D. I. Small-angle X-ray and neutron scattering. Nature Reviews Methods Primers 2021, 1, 70. 
(34) Narayanan, T.; Sztucki, M.; Van Vaerenbergh, P.; Léonardon, J.; Gorini, J.; Claustre, L.; Sever, F.; Morse, J.; Boesecke, P. A Multipurpose Instrument for Time-Resolved Ultra-Small-Angle and Coherent X-ray Scattering. J Appl Crystallogr 2018, 51, 15111524 .

(35) Glinka, C.; Bleuel, M.; Tsai, P.; Zákutná, D.; Honecker, D.; Dresen, D.; Mees, F.; Disch, S. Sub-Millisecond Time-Resolved Small-Angle Neutron Scattering Measurements at NIST. J Appl Cryst 2020, 53, 598-604.

(36) Zákutná, D.; Graef, K.; Dresen, D.; Porcar, L.; Honecker, D.; Disch, S. In Situ Magnetorheological SANS Setup at Institut Laue-Langevin. Colloid Polym Sci 2021, 299, 281-288.

(37) Morozov, K. I.; Mirzae, Y.; Kenneth, O.; Leshansky, A. M. Dynamics of arbitrary shaped propellers driven by a rotating magnetic field. Phys. Rev. Fluids 2017, 2, 044202 .

(38) Schamel, D.; Pfeifer, M.; Gibbs, J. G.; Miksch, B.; Mark, A. G.; Fischer, P. Chiral Colloidal Molecules And Observation of The Propeller Effect. J. Am. Chem. Soc. 2013, 135, 12353-12359.

(39) Morozov, K. I.; Leshansky, A. M. Towards Focusing of a Swarm of Magnetic Micro/Nanomotors. Phys. Chem. Chem. Phys. 2020, 22, 16407. 


\section{TOC Graphic}

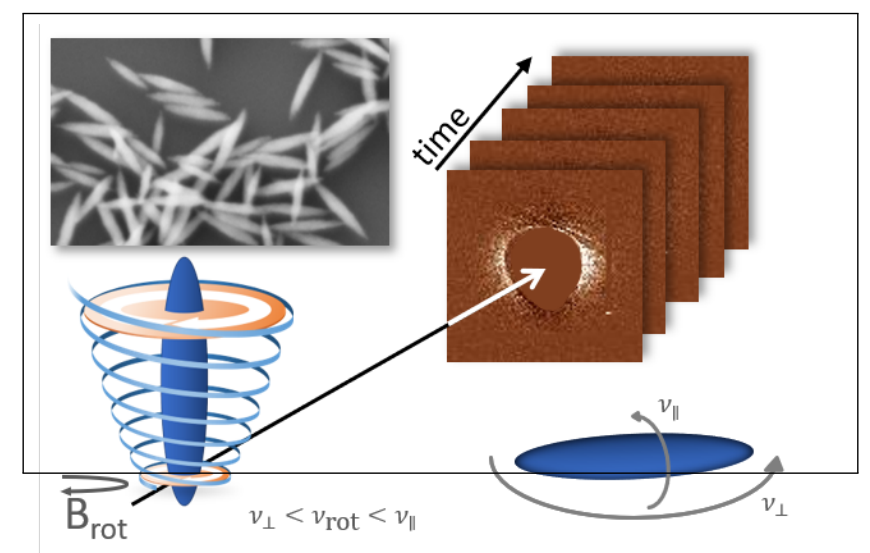

